\title{
Conductance length autocorrelation in quasi one-dimensional disordered wires
}

\author{
Klaus Frahm* and Axel Müller-Groeling ${ }^{\dagger}$ \\ Service de Physique de l'État condensé, CEA Saclay, 91191 Gif-sur-Yvette, France.
}

\begin{abstract}
Employing techniques recently developed in the context of the Fokker-Planck approach to electron transport in disordered systems we calculate the conductance length correlation function $\langle\delta g(L) \delta g(L+\Delta L)\rangle$ for quasi 1d wires. Our result is valid for arbitrary lengths $L$ and $\Delta L$. In the metallic limit the correlation function is given by a squared Lorentzian. In the localized regime it decays exponentially in both $L$ and $\Delta L$. The correlation length is proportional to $L$ in the metallic regime and saturates at a value approximately given by the localization length $\xi$ as $L \gg \xi$.

72.15.Rn, 05.60.+w, 02.50.-r
\end{abstract}

Typeset using REVTEX

*Present address: Laboratoire de Physique Quantique, Université Paul Sabatier, F-31062 Toulouse, France

†Present address: Max-Planck-Institut für Kernphysik, D-69029 Heidelberg, Germany 


\section{INTRODUCTION}

In the last fifteen years two powerful approaches to calculate the electron transport properties of quasi 1d disordered wires have been developed: a method based on the nonlinear $\sigma$ model by Efetov and Larkin [1] and a Fokker-Planck approach by Dorokhov [2], Mello, Pereyra and Kumar [3]. Both methods are nonperturbative in the sense that they do not assume that the system size is small compared with the localization length $\xi$. Therefore the full range from the metallic to the localized regime can be described within these two frameworks. Very recently it has been shown [4] that both approaches are in fact equivalent, despite their considerable technical dissimilarity. Apart from the important conceptual point of unifying our theoretical understanding of quasi $1 \mathrm{~d}$ wires this proof of equivalence enables us to choose among the available techniques the more suitable one for a given problem. Combining the results obtained within both approaches, our knowledge about quasi $1 \mathrm{~d}$ transport is very advanced, some would say almost complete. We mention only a few important facts: Both the conductance $g$ and its variance $\operatorname{var}(g)$ have been calculated for arbitrary system length and for all three symmetry classes [5, 4. The probability distribution function for the generalized eigenvalues of the transfer matrix is known exactly in the unitary case [6] and the corresponding $n$-point correlation functions are also under control [7]. However, in spite of all this progress, there are still a few unsolved problems. One of the probably most important questions concerns the magnetic field correlation function $\langle\delta g(L, B) \delta g(L, B+\Delta B)\rangle$. This quantity cannot be formulated within the so-called "minimal" $\sigma$ model, i.e. the supermatrix space necessary to describe this correlation function is larger than in previous nonperturbative calculations. The technical difficulties to treat larger $\sigma$ models exactly have so far been insurmountable. In the Fokker-Planck approach, on the other hand, the question is how to incorporate the magnetic field in the theoretical formulation. This more conceptual problem also still awaits a solution.

In this paper we calculate the conductance length correlation function $\langle\delta g(L) \delta g(L+\Delta L)\rangle$ for all $L$ and $\Delta L$ using the Fokker-Planck technique. In this case the situation is more favourable than for the magnetic field correlation function since the system length - unlike the magnetic field — is the fundamental parameter of the whole approach. Therefore it is possible to perform an exact calculation of a quantity that cannot be formulated within the framework of the minimal $\sigma$ model. We briefly summarize our results: In the metallic limit $L, \Delta L \ll \xi$ the length correlation function is given by a squared Lorentzian, while it decays exponentially as a function of both $L$ and $\Delta L$ in the localized regime $L, \Delta L \gg \xi$. As $L$ increases from the metallic to the localized limit the correlation length in $\Delta L$, which is proportional to $L$ for $L \ll \xi$, saturates at a constant value equal to $\xi$. This explicitly confirms the view that a strongly localized wire is composed of independently fluctuating segments of size $\xi$. In a recent paper [8], S. Feng, J.-L. Pichard and F. Zeng have argued that, in the localized regime, the conductance fluctuations induced by the motion of a single impurity should be universal, $\left\langle\left[\ln g / g^{\prime}\right]^{2}\right\rangle \approx 1$. Here, $g$ and $g^{\prime}$ are the conductances before and after the impurity has moved, respectively. The main qualitative assumption necessary to arrive at this conclusion was precisely the decomposition into segments, which is rigorously derived in our paper. Therefore, the universality of the conductance fluctuations in the localized regime can now be regarded as analytically established in quasi 1d wires. The arguments in [8], however, go beyond the quasi 1d case and suggest, supported by numerical simulations, 
that this universality extends also to $2 \mathrm{~d}$ and $3 \mathrm{~d}$.

The conductance length autocorrelation function is in principle accessible by experiments in mesoscopic nanostructures. For this, one has to fabricate a "quasi one-dimensional" quantum-wire with several contacts at discrete distances $L_{j}$. Using such a geometry, one can measure the conductance as a function of $L_{j}$ (and of the magnetic field $B$ ), where the disorder realization is identical for the common parts of the wire. One has to take into account, however, that in these multi-lead devices phase coherent scattering in the attached contact arms typically influences the experimental result. As usual, the statistical average necessary to calculate the mean conductance or the length autocorrelation function may be replaced by an average over a suitable range of the magnetic field.

Our paper is organized as follows. After collecting a few important known results concerning the Fokker-Planck approach in section II, we develop the technical framework for the length correlation function of an arbitrary linear statistic in section III. In section IV we derive a (complex) expression for the special case of the conductance length correlation function. Sections V,VI and VII are devoted to the metallic, localized and crossover regimes, respectively. In section VIII we give a short summary and make some concluding remarks. Considerations of a purely technical nature have been deferred to appendices A to E.

\section{KNOWN RESULTS AND BASIC TERMINOLOGY}

In the Fokker-Planck approach [2,3,9, 11] the transmission properties of a quasi 1d wire with $N$ conducting channels and length $L$ are characterized by $N$ generalized eigenvalues $\lambda_{1} \geq 0(i=1, \ldots, N)$. They parametrize the radial part [3, 12] of the transfer matrix. With $T_{i}=\left(1+\lambda_{i}\right)^{-1}$ the transmission coefficients, the conductance $g$ is given by the Landauer formula

$$
g=\sum_{i=1}^{N} T_{i}=\sum_{i=1}^{N} \frac{1}{1+\lambda_{i}}
$$

The multiplicative combination law for the transfer matrix yields the following FokkerPlanck equation [2, 3, 11] for the probability distribution $p(\hat{\lambda}, t)$ of the wire,

$$
\partial_{t} p(\hat{\lambda}, t)=\Delta p(\hat{\lambda}, t)
$$

Here, $\hat{\lambda}=\left(\lambda_{1}, \ldots, \lambda_{N}\right)$ is the collection of eigenvalues $\lambda_{i}, t=L / 2 \xi$ the length of the wire measured in units of (twice) the localization length $\xi$, and $\Delta$ the radial part of the Laplacian on the transfer matrix space,

$$
\begin{aligned}
\Delta & =4 \sum_{i} \partial_{\lambda_{i}} \lambda_{i}\left(1+\lambda_{i}\right) J(\hat{\lambda}) \partial_{\lambda_{i}} J^{-1}(\hat{\lambda}), \\
J(\hat{\lambda}) & =\prod_{i>j}\left|\lambda_{i}-\lambda_{j}\right|^{\beta} .
\end{aligned}
$$

We have $\xi=(\beta N+2-\beta) l$, where $\beta=1,2,4$ corresponds to the orthogonal, unitary, and symplectic symmetry class, respectively, and $l$ is the elastic mean free path. The usual initial condition for (2) is given by $p(\hat{\lambda}, 0)=\delta(\hat{\lambda})$, corresponding to ideal transmission $T_{i}=1$ for a wire of zero length. In the unitary case (to which we restrict our attention 
in this paper) the Fokker-Planck equation (2) has been solved exactly [6] by means of a Sutherland-transformation. Subsequently, the $n$-point correlation functions of the $\lambda_{i}$ (at a fixed value of the length $L$ ) have been calculated [7] for arbitrary $N$ and $L$. In the following we summarize some of the results in [6,7] for further reference.

The probability distribution $p(\hat{\lambda}, t)$ can be expressed [6] in terms of a certain "manybody" Green's function $G(\hat{\mu}, \hat{\lambda} ; t)$

$$
p(\hat{\lambda}, t)=\int d^{N} \hat{\mu} G(\hat{\mu}, \hat{\lambda} ; t) p(\hat{\mu}, 0)=\lim _{\hat{\mu} \rightarrow 0} G(\hat{\mu}, \hat{\lambda} ; t)
$$

This Green's function is given by

$$
G(\hat{\mu}, \hat{\lambda} ; t)=\frac{1}{N !} \frac{\rho(\hat{\lambda})}{\rho(\hat{\mu})} \operatorname{det}\left(\left(g\left(\mu_{i}, \lambda_{j} ; t\right)\right) e^{C_{N} t}\right.
$$

where

$$
\rho(\hat{\lambda})=\prod_{i>j}\left(\lambda_{i}-\lambda_{j}\right) \quad, \quad C_{N}=-\sum_{n=0}^{N-1} \varepsilon_{n} \quad, \quad \varepsilon_{n}=(1+2 n)^{2}
$$

and $g(\mu, \lambda ; t)$ is a "one-body" Green's function corresponding to the differential operator

$$
D(\lambda)=-\left(4 \partial_{\lambda} \lambda(1+\lambda) \partial_{\lambda}+1\right) .
$$

We have

$$
\partial_{t} g(\mu, \lambda ; t)=-D(\lambda) g(\mu, \lambda ; t)=-D(\mu) g(\mu, \lambda ; t) \quad, \quad g(\mu, \lambda ; 0)=\delta(\mu-\lambda)
$$

The operator $D(\lambda)$ has a continuous set of eigenfunctions

$$
\psi_{k}(\lambda)=P_{\frac{1}{2}(i k-1)}(1+2 \lambda)=F\left(\frac{1}{2}+i \frac{k}{2}, \frac{1}{2}-i \frac{k}{2} ; 1 ;-\lambda\right)
$$

with eigenvalues $k^{2}$, i.e. $D(\lambda) \psi_{k}(\lambda)=k^{2} \psi_{k}(\lambda)$. Here, $P_{\frac{1}{2}(i k-1)}$ denotes the generalized Legendre function, which can be expressed in terms of the hypergeometric function $F(a, b ; c ; z)$ as indicated above [13]. The expansion of $g(\mu, \lambda ; t)$ in terms of these eigenfunctions is given by $[6]$

$$
g(\mu, \lambda ; t)=\int_{0}^{\infty} d k \frac{k}{2} \tanh \left(\frac{\pi k}{2}\right) \psi_{k}(\mu) \psi_{k}(\lambda) e^{-k^{2} t}
$$

Using (6) and (11) the limit in (5) can be evaluated [6]. The resulting expression can be rewritten [7] in the very useful form

$$
p(\hat{\lambda}, t)=\frac{1}{N !} \operatorname{det}\left(Q_{n-1}\left(\lambda_{j} ; t\right)\right) \operatorname{det}\left(h_{m-1}\left(\lambda_{i} ; t\right)\right)
$$

where $(n, m=0,1, \ldots, N-1)$

$$
\begin{aligned}
Q_{n}(\lambda ; t) & =P_{n}(1+2 \lambda) e^{-\varepsilon_{n} t} \\
h_{m}(\lambda ; t) & =\int_{0}^{\infty} d k \frac{k}{2} \tanh \left(\frac{\pi k}{2}\right) L_{m}\left(k^{2}\right) \psi_{k}(\lambda) e^{-k^{2} t} \\
L_{m}(z) & =\prod_{l=0,(l \neq m)}^{N-1} \frac{z-\left(-\varepsilon_{l}\right)}{\left(-\varepsilon_{m}\right)-\left(-\varepsilon_{l}\right)}
\end{aligned}
$$


The Legendre polynomial $P_{n}(1+2 \lambda)$ in (13) is also an eigenfunction of $D(\lambda)$. The corresponding eignevalue is $-\varepsilon_{n}$. The $P_{n}(1+2 \lambda)$ do not contribute in the expansion (11) because they are not normalizable in the range of integration $\lambda \geq 0$. The advantage of the representation (12) is due to the biorthogonality relation [0]

$$
\int_{0}^{\infty} d \lambda Q_{n}(\lambda ; t) h_{m}(\lambda ; t)=\delta_{n m}
$$

which was the key to calculating the $n$-point correlation functions [0].

In the following, we will also need the two properties

$$
\begin{aligned}
\int_{0}^{\infty} d \mu h_{m}(\mu ; t) g(\mu, \lambda ; \Delta t) & =h_{m}(\lambda ; t+\Delta t), \\
\int_{0}^{\infty} d \lambda g(\mu, \lambda ; \Delta t) Q_{n}(\lambda ; t+\Delta t) & =Q_{n}(\mu ; t) .
\end{aligned}
$$

These identities can be verified using (9), (14) and the fact that $\partial_{t} Q_{n}(\lambda ; t)=D(\lambda) Q_{n}(\lambda ; t)$ $(\operatorname{see}(13))$.

\section{DENSITY-DENSITY CORRELATION FUNCTION FOR THE EIGENVALUES}

We are finally interested in calculating the conductance length correlation function $\langle\delta g(L) \delta g(L+\Delta L)\rangle$. To this end we need to know the joint probability density function of finding a certain fixed set of eigenvalues $\hat{\mu}$ at $t$ and another fixed set $\hat{\lambda}$ at $t+\Delta t$. Obviously, this density is given by

$$
p_{2}(\hat{\mu}, t ; \hat{\lambda}, t+\Delta t)=p(\hat{\mu} ; t) G(\hat{\mu}, \hat{\lambda} ; \Delta t)
$$

since the conditional probability density of finding a transition $\hat{\mu} \rightarrow \hat{\lambda}$ in a length intervall $\Delta t$ is precisely given by the propagator $G(\hat{\mu}, \hat{\lambda} ; \Delta t)$.

We are now going to formulate a slightly more general problem than the one we wish to solve in the end. Let $A_{1,2}=\sum_{i} a_{1,2}\left(\lambda_{i}\right)$ be two arbitrary linear statistics of the eigenvalues $\lambda_{i}$. Then the length correlation function of these two quantities can be written as

$$
\left\langle A_{1}(t) A_{2}(t+\Delta t)\right\rangle=\int_{0}^{\infty} d \lambda_{1} \int_{0}^{\infty} d \mu_{1} a_{1}\left(\lambda_{1}\right) a_{2}\left(\mu_{1}\right) R_{1,1}\left(\mu_{1}, t ; \lambda_{1} ; t+\Delta t\right) .
$$

The function $R_{1,1}\left(\mu_{1}, t ; \lambda_{1} ; t+\Delta t\right)$ is the probability density of finding one eigenvalue equal to $\mu_{1}$ at $t$ and one eigenvalue equal two $\lambda_{1}$ at $t+\Delta t$. It arises from the joint probability density (19) upon integrating out all the remaining eigenvalues,

$$
R_{1,1}\left(\mu_{1}, t ; \lambda_{1} ; t+\Delta t\right)=N^{2} \int d \mu_{2} \ldots d \mu_{N} d \lambda_{2} \ldots d \lambda_{N} p_{2}(\hat{\mu}, t ; \hat{\lambda}, t+\Delta t)
$$

Using the fact that $\operatorname{det}\left(Q_{n-1}\left(\lambda_{j} ; t\right)\right)=$ const. $\rho(\hat{\lambda}) e^{C_{N} t}$ and with the help of (6), (12), and (19) we can reexpress $p_{2}$ as

$$
p_{2}(\hat{\mu}, t ; \hat{\lambda}, t+\Delta t)=\frac{1}{N !^{2}} \operatorname{det}\left(h_{m-1}\left(\mu_{i} ; t\right)\right) \operatorname{det}\left(g\left(\mu_{i}, \lambda_{j} ; \Delta t\right) \operatorname{det}\left(Q_{n-1}\left(\lambda_{j} ; t+\Delta t\right)\right)\right.
$$


Rather than calculating all the integrations in (21) explicitly, we will employ a generating functional [14 to calculate the density (21): Let $u_{1,2}(\lambda)$ be arbitrary functions of the eigenvalue $\lambda$. Following [14] we define the functional

$$
\begin{aligned}
L\left[u_{1}, u_{2}\right] & =\left\langle\prod_{i} u_{1}\left(\lambda_{i}(t)\right) \prod_{j} u_{2}\left(\lambda_{j}(t+\Delta t)\right)\right\rangle \\
& =\int d^{N} \hat{\mu} \int d^{N} \hat{\lambda} \prod_{i}\left(u_{1}\left(\mu_{i}\right) u_{2}\left(\lambda_{i}\right)\right) p_{2}(\hat{\mu}, t ; \hat{\lambda}, t+\Delta t)
\end{aligned}
$$

so that the correlation function (20) can be expressed as

$$
\left\langle A_{1}(t) A_{2}(t+\Delta t)\right\rangle=\left.\partial_{z_{1}} \partial_{z_{2}} L\left[1+z_{1} a_{1}, 1+z_{2} a_{2}\right]\right|_{z_{1,2}=0}
$$

Inserting (22) in (23) we find after some algebra involving transformations of the various determinants

$$
L\left[u_{1}, u_{2}\right]=\operatorname{det}\left(M_{m n}\left[u_{1}, u_{2}\right]\right) \quad,
$$

with the $N \times N$-matrix

$$
M_{m n}\left[u_{1}, u_{2}\right]=\int_{0}^{\infty} d \mu \int_{0}^{\infty} d \lambda h_{m}(\mu ; t) g(\mu, \lambda ; \Delta t) Q_{n}(\lambda ; t+\Delta) u_{1}(\mu) u_{2}(\lambda)
$$

depending linearly on $u_{1}$ and $u_{2}$. To calculate the derivatives in (24) we notice that due to (16)-(18) we have $M_{m n}[1,1]=\delta_{n m}$ so that (in matrix notation)

$$
\begin{aligned}
M\left[1+z_{1} a_{1}, 1+z_{2} a_{2}\right] & =1+z_{1} M\left[a_{1}, 1\right]+z_{2} M\left[1, a_{2}\right]+z_{1} z_{2} M\left[a_{1}, a_{2}\right] \\
& \equiv 1+X .
\end{aligned}
$$

To expand the determinant in (25) we employ the relation

$$
\operatorname{det}(1+X)=\exp (\operatorname{tr} \ln (1+X))=1+\operatorname{tr}(X)+\frac{1}{2}\left(\operatorname{tr}(X)^{2}-\operatorname{tr}\left(X^{2}\right)\right)+\cdots \quad .
$$

Finally, using (25), (27), and (28) in (24) we can perform the derivatives with respect to the auxiliary variables $z_{1}$ and $z_{2}$ and arrive at

$$
\left\langle A_{1}(t) A_{2}(t+\Delta t)\right\rangle=\operatorname{tr}\left(M\left[a_{1}, a_{2}\right]\right)+\operatorname{tr}\left(M\left[a_{1}, 1\right]\right) \operatorname{tr}\left(M\left[1, a_{2}\right]\right)-\operatorname{tr}\left(M\left[a_{1}, 1\right] M\left[1, a_{2}\right]\right) .
$$

This result can now be straightforwardly compared with the expression (20) to identify the two-point density $R_{1,1}(\mu, t ; \lambda, t+\Delta t)$. To write $R_{1,1}$ in a compact and appropriate form we define the function

$$
K_{N}\left(\lambda, t_{1} ; \mu, t_{2}\right)=\sum_{m=0}^{N-1} Q_{m}\left(\lambda ; t_{1}\right) h_{m}\left(\mu ; t_{2}\right)
$$

in terms of which the one-point density $R_{1}(\mu, t)$ reads [7] 


$$
R_{1}(\mu ; t)=K_{N}(\mu, t ; \mu, t) .
$$

This quantity occurs in the calculation of simple averages of a linear statistic such as

$$
\left\langle A_{1}(t)\right\rangle=\left.\partial_{z_{1}} L\left[1+z_{1} a_{1}, 1\right]\right|_{z_{1}=0}=\operatorname{tr}\left(M\left[a_{1}, 1\right]\right)=\int_{0}^{\infty} d \mu a_{1}(\mu) R_{1}(\mu ; t) .
$$

With the definitions (30) and (31) and taking into account the relations (16)-(18) the comparison between (20) and (29) results in

$$
\begin{aligned}
R_{1,1}(\mu, t ; \lambda ; t+\Delta t)= & R_{1}(\mu ; t) R_{1}(\lambda ; t+\Delta t) \\
& +K_{N}(\lambda, t+\Delta t ; \mu, t)\left[g(\mu, \lambda ; \Delta t)-K_{N}(\mu, t ; \lambda, t+\Delta t)\right] .
\end{aligned}
$$

To summarize the result of this section, the correlator of $A_{1}(t)$ and $A_{2}(t+\Delta t)$ is given by

$$
\begin{aligned}
\left\langle\delta A_{1}(t) \delta A_{2}(t+\Delta t)\right\rangle & =\left\langle A_{1}(t) A_{2}(t+\Delta t)\right\rangle-\left\langle A_{1}(t)\right\rangle\left\langle A_{2}(t+\Delta t)\right\rangle \\
& =\int_{0}^{\infty} d \lambda \int_{0}^{\infty} d \mu a_{1}(\lambda) a_{2}(\mu) S(\mu, t ; \lambda ; t+\Delta t),
\end{aligned}
$$

where we have introduced the density-density correlation function for the generalized eigenvalues of the transfer matrix,

$$
S(\mu, t ; \lambda ; t+\Delta t)=K_{N}(\lambda, t+\Delta t ; \mu, t)\left[g(\mu, \lambda ; \Delta t)-K_{N}(\mu, t ; \lambda, t+\Delta t)\right] .
$$

In the next section we derive an explicit expression for the conductance length correlation function starting from (34) and (35).

\section{CONDUNCTANCE LENGTH CORRELATION FUNCTION}

The correlator $\langle\delta g(t) \delta g(t+\Delta t)\rangle$ is a special case of (34), obtained by choosing $a_{1,2}(\lambda)=$ $(1+\lambda)^{-1}$,

$$
\langle\delta g(t) \delta g(t+\Delta t)\rangle=\int_{0}^{\infty} d \lambda \int_{0}^{\infty} d \mu \frac{1}{1+\mu} \frac{1}{1+\lambda} S(\mu, t ; \lambda ; t+\Delta t) .
$$

The evaluation of this expression is a little involved and makes use of a few mathematical identities, which we state as we go along. To begin with, inserting (35) in (36) we have $\langle\delta g(t) \delta g(t+\Delta t)\rangle=C_{1}+C_{2}$, where

$$
\begin{aligned}
& C_{1}=\int_{0}^{\infty} d \lambda d \mu \frac{1}{1+\lambda} \frac{1}{1+\mu} K_{N}(\lambda, t+\Delta t ; \mu, t) g(\mu, \lambda ; \Delta t) \\
& C_{2}=-\int_{0}^{\infty} d \lambda d \mu \frac{1}{1+\lambda} \frac{1}{1+\mu} K_{N}(\lambda, t+\Delta t ; \mu, t) K_{N}(\mu, t ; \lambda, t+\Delta t) .
\end{aligned}
$$

The second contribution, $C_{2}$, is the easier one to calculate. Replacing the $K_{N}$ in (38) by the r.h.s. of (30) one realizes that the relevant remaining integral is given by

$$
\int d \mu \frac{1}{1+\mu} h_{m}(\mu, t) Q_{m^{\prime}}(\mu, t)
$$


Using the expansion (see appendix A)

$$
\begin{aligned}
\frac{1}{1+\lambda} Q_{m}(1+2 \lambda) & =(-1)^{m} e^{-\varepsilon_{m} t}\left(\frac{1}{1+\lambda}+\sum_{l=0}^{m-1} a_{m l} e^{\varepsilon_{l} t} Q_{l}(1+2 \lambda)\right) \\
a_{m l} & =2(-1)^{l+1}(1+2 l) \sum_{\nu=l+1}^{m} \frac{1}{\nu} \quad\left(a_{m l}=0 \text { for } l \geq m\right)
\end{aligned}
$$

the abbreviation

$$
F_{m}(t)=\int_{0}^{\infty} d \mu \frac{1}{1+\mu} h_{m}(\mu ; t)
$$

and the biorthogonality relation (16) it is not difficult to see that

$$
\int_{0}^{\infty} d \mu \frac{1}{1+\mu} h_{m}(\mu ; t) Q_{l}(\mu ; t)=(-1)^{l} e^{-\varepsilon_{l} t} F_{m}(t)+(-1)^{l} a_{l m} e^{\left(\varepsilon_{m}-\varepsilon_{l}\right) t},
$$

and hence

$$
\begin{aligned}
C_{2}= & -\left(\sum_{m=0}^{N-1}(-1)^{m} e^{-\varepsilon_{m}(t+\Delta t)} F_{m}(t)\right)\left(\sum_{l=0}^{N-1}(-1)^{l} e^{-\varepsilon_{l} t} F_{l}(t+\Delta t)\right) \\
& -\sum_{m, l=0}^{N-1}(-1)^{m+l} a_{m l}\left(F_{m}(t+\Delta t) e^{-\varepsilon_{l} \Delta t-\varepsilon_{m} t}+F_{m}(t) e^{-\varepsilon_{m}(t+\Delta t)+\varepsilon_{l} \Delta t}\right) .
\end{aligned}
$$

The slightly more complicated contribution $C_{1}$ can be evaluated as follows. We again replace $K_{N}$ by the r.h.s. of (30) and employ the decomposition (40), so that $C_{1}=C_{3}+C_{4}$ with

$$
\begin{aligned}
& C_{3}=\sum_{m=0}^{N-1}(-1)^{m} e^{-\varepsilon_{m}(t+\Delta t)} \int_{0}^{\infty} d \lambda d \mu \frac{1}{1+\lambda} \frac{1}{1+\mu} h_{m}(\mu ; t) g(\mu, \lambda ; \Delta t), \\
& C_{4}=\sum_{m, l=0}^{N-1}(-1)^{m} e^{\left(\varepsilon_{l}-\varepsilon_{m}\right)(t+\Delta t)} a_{m l} \int_{0}^{\infty} d \mu \frac{1}{1+\mu} h_{m}(\mu ; t) Q_{l}(\mu ; t)
\end{aligned}
$$

To simplify (46) we have taken advantage of (18). Using the decomposition (40) in (46) and recalling (16) we get

$$
C_{4}=\sum_{m, l=0}^{N-1}(-1)^{m+l} a_{m l} e^{\varepsilon_{l} \Delta t-\varepsilon_{m}(t+\Delta t)} F_{m}(t) .
$$

Next, to evaluate $C_{3}$ we insert the relations (11) and (14) in (45) to get

$$
\begin{aligned}
C_{3}= & \sum_{m=0}^{N-1} \int_{0}^{\infty} d k_{1} \frac{k_{1}}{2} \tanh \left(\frac{\pi k_{1}}{2}\right) L_{m}\left(k_{1}^{2}\right)(-1)^{m} e^{-\varepsilon_{m}(t+\Delta t)} e^{-k_{1}^{2} t} \\
& \int_{0}^{\infty} d k_{2} \frac{k_{2}}{2} \tanh \left(\frac{\pi k_{2}}{2}\right) e^{-k_{2}^{2} \Delta t} \int_{0}^{\infty} d \mu \frac{1}{1+\mu} \psi_{k_{1}}(\mu) \psi_{k_{2}}(\mu) \int_{0}^{\infty} d \lambda \frac{1}{1+\lambda} \psi_{k_{2}}(\lambda)
\end{aligned}
$$

For further progress we need the intgral 


$$
\int_{0}^{\infty} d \lambda(1+\lambda)^{-\alpha} \psi_{k}(\lambda)=\frac{\Gamma\left(-\frac{1}{2}+\alpha-i \frac{k}{2}\right) \Gamma\left(-\frac{1}{2}+\alpha+i \frac{k}{2}\right)}{\Gamma(\alpha)^{2}}
$$

as derived in appendix $\mathrm{B}$ for arbitrary complex $\alpha$ with $\operatorname{Re}(\alpha) \geq 1 / 2$ and

$$
\begin{aligned}
L_{m}\left(k^{2}\right) & =\frac{(-1)^{m} 4(1+2 m)}{k^{2}+\varepsilon_{m}} \frac{a(N, m, k)}{\Gamma\left(\frac{1}{2}-i \frac{k}{2}\right) \Gamma\left(\frac{1}{2}+i \frac{k}{2}\right)}, \\
a(N, m, k) & =\frac{\Gamma\left(N+\frac{1}{2}+i \frac{k}{2}\right) \Gamma\left(N+\frac{1}{2}-i \frac{k}{2}\right)}{\Gamma(N-m) \Gamma(N+m+1)},
\end{aligned}
$$

which follows by a straightforward calculation from (15). Furthermore we notice that the function $F_{m}(t)$ defined in (42) can be written by virtue of (14), (49) and (50) as

$$
F_{m}(t)=(-1)^{m} 4(1+2 m) \int_{0}^{\infty} d k \frac{k}{2} \tanh \left(\frac{\pi k}{2}\right) \frac{a(N, m, k)}{k^{2}+\varepsilon_{m}} e^{-k^{2} t} .
$$

After all these steps $\langle\delta g(t) \delta g(t+\Delta t)\rangle$ is expressed as the sum of $C_{2}, C_{3}$, and $C_{4}$ as given in (44), (48), and (47), respectively, after taking into account the relations (49)-(51). Obviously, a simplification of the notation is called for. Let us therefore define the symbol $[\ldots]_{t}^{(k, m)}$ by

$$
[f(k, m)]_{t}^{(k, m)}=\sum_{m=0}^{N-1} \int_{0}^{\infty} d k \frac{k}{2} \tanh \left(\frac{\pi k}{2}\right) \frac{4(1+2 m) a(N, m, k)}{k^{2}+\varepsilon_{m}}\{f(k, m)\} e^{-\left(k^{2}+\varepsilon_{m}\right) t}
$$

with $f(k, m)$ an arbitrary function of $k$ and $m$. With this notation the conductance length correlation function is given by

$$
\begin{aligned}
\langle\delta g(t) \delta g(t+\Delta t)\rangle= & {\left[S_{1}(k, \Delta t) e^{-\varepsilon_{m} \Delta t}\right]_{t}^{(k, m)}-\left[S_{2}(m, \Delta t) e^{-k^{2} \Delta t}\right]_{t}^{(k, m)} } \\
& -\left[e^{-\varepsilon_{m} \Delta t}\right]_{t}^{(k, m)}\left[e^{-k^{2} \Delta t}\right]_{t}^{(k, m)}
\end{aligned}
$$

with

$$
\begin{aligned}
S_{1}(k, \Delta t) & =\frac{1}{\Gamma\left(\frac{1}{2}-i \frac{k}{2}\right) \Gamma\left(\frac{1}{2}+i \frac{k}{2}\right)} \int_{0}^{\infty} d \mu \frac{\psi_{k}(\mu)}{1+\mu} \int_{0}^{\infty} d \lambda \frac{g(\mu, \lambda ; \Delta t)}{1+\lambda} \\
& =\int_{0}^{\infty} d \tilde{k} \frac{\tilde{k}}{2} \tanh \left(\frac{\pi \hat{k}}{2}\right) \frac{\Gamma\left(\frac{1}{2}-i \frac{\tilde{k}}{2}\right) \Gamma\left(\frac{1}{2}+i \frac{\tilde{k}}{2}\right)}{\Gamma\left(\frac{1}{2}-i \frac{k}{2}\right) \Gamma\left(\frac{1}{2}+i \frac{k}{2}\right)} e^{-\tilde{k}^{2} \Delta t} I(k, \tilde{k}), \\
S_{2}(m, \Delta t) & =\sum_{l=0}^{m-1} a_{m l}(-1)^{l} e^{-\varepsilon_{l} \Delta t}=-2 \sum_{\nu=1}^{m} \frac{1}{\nu} \sum_{l=0}^{\nu-1}(1+2 l) e^{-\varepsilon_{l} \Delta t}
\end{aligned}
$$

and the abbreviation

$$
I(k, \tilde{k})=\int_{0}^{\infty} d \mu \frac{1}{1+\mu} \psi_{k}(\mu) \psi_{\tilde{k}}(\mu) .
$$

In general, the summations and integrations in (53) have to be carried out numerically. The most difficult numerical task is the evaluation of (56). In appendix $\mathrm{C}$ we derive an expression 
for $I(k, \tilde{k})$ which allows for an efficient numerical treatment. For completeness, we mention that the average conductance can be calculated from (32) and is given by [7]

$$
\langle g(t)\rangle=[1]_{t}^{(k, m)} .
$$

In the limiting cases of a metallic wire $(N \rightarrow \infty, t \ll 1)$ and of a localized wire $(t \gg 1)$ further analytical progress in the calculation of the conductance length correlation function (53) is possible. The next two sections deal with these two limits, respectively.

\section{METALLIC REGIME}

In this section we show that in the metallic limit $t \ll 1$ and for large channel numbers $N \rightarrow \infty$ the conductance length correlation function is given by a squared Lorentzian, i.e.

$$
\langle\delta g(t) \delta g(t+\Delta t)\rangle=\frac{1}{15} \frac{1}{\left(1+\frac{\Delta t}{t}\right)^{2}}+\mathcal{O}(t)
$$

While this result is rather simple its derivation requires considerable effort. We begin by observing that for $N \rightarrow \infty$ the coefficient $a(N, m, k)$ (see(50)) tends to unity [13] so that the following decomposition rule for the symbol $[\ldots]_{t}^{(k, m)}$ holds:

$$
\left(-\partial_{t}\right)\left[f_{1}(k) f_{2}(m)\right]_{t}^{(k, m)}=\left[f_{1}(k)\right]_{t}^{(k)}\left[f_{2}(m)\right]_{t}^{(m)}
$$

Here, $f_{1}(k)$ and $f_{2}(m)$ are arbitrary functions and we have introduced the independent $k^{-}$ and $m$-"averages":

$$
\begin{aligned}
{\left[f_{1}(k)\right]_{t}^{(k)} } & =\int_{0}^{\infty} d k k \tanh \left(\frac{\pi k}{2}\right) e^{-k^{2} t}\left\{f_{1}(k)\right\} \\
{\left[f_{2}(m)\right]_{t}^{(m)} } & =\sum_{m=0}^{\infty} 2(1+2 m) e^{-\varepsilon_{m} t}\left\{f_{2}(m)\right\}
\end{aligned}
$$

Our derivation of (58) relies essentially on the small $t$-behavior of the quantities $[1]_{t}^{(k)}$ and $[1]_{t}^{(m)}$, which will shortly be seen to become important,

$$
\begin{aligned}
& {[1]_{t}^{(k)}=\frac{1}{2 t}\left(1-\frac{1}{3} t+\frac{7}{30} t^{2}\right)+\mathcal{O}\left(t^{2}\right)} \\
& {[1]_{t}^{(m)}=\frac{1}{2 t}\left(1+\frac{1}{3} t+\frac{7}{30} t^{2}\right)+\mathcal{O}\left(t^{2}\right)+\mathcal{O}\left(e^{-\pi^{2} /(4 t)}\right)}
\end{aligned}
$$

These expansions are derived to all orders in $t$ in appendix D. In (63) we have also indicated the presence of non-analytical contributions.

Let us first consider the case $\Delta t=0$, i.e. the case of universal conductance fluctuations. Using (9), (11), (54), and (55) one can show that $S_{1}(k, 0)=\frac{1}{4}\left(1+k^{2}\right)$ and $S_{2}(m, 0)=$ $-m(m-1)=\frac{1}{4}\left(1-\varepsilon_{m}\right)$. Hence we have from (53)

$$
\left\langle\delta g^{2}(t)\right\rangle=\frac{1}{4}\left[k^{2}+\varepsilon_{m}\right]_{t}^{(k, m)}-\left([1]_{t}^{(k, m)}\right)^{2}=\frac{1}{4}[1]_{t}^{(k)}[1]_{t}^{(m)}-\left([1]_{t}^{(k, m)}\right)^{2}
$$


With the help of the expansions (62), (63) and using (59) we obtain

$$
\begin{aligned}
{[1]_{t}^{(k)}[1]_{t}^{(m)} } & =\frac{1}{4 t^{2}}+\frac{4}{45}+\mathcal{O}\left(t^{2}\right) \\
{[1]_{t}^{(k, m)} } & =-\int d t\left([1]_{t}^{(k)}[1]_{t}^{(m)}\right)=\frac{1}{4 t}-\frac{4}{45} t+\mathcal{O}\left(t^{3}\right) .
\end{aligned}
$$

(We omit a formal proof that the integration constant in (66) is zero. A nonzero integration constant contradicts the fact that $\left\langle\delta g(t)^{2}\right\rangle=\mathcal{O}(1)$ in the metallic regime). Inserting (65) and (66) in (64) we find the well-known result

$$
\left\langle\delta g^{2}(t)\right\rangle=\frac{1}{15}+\mathcal{O}\left(t^{2}\right)
$$

As a side remark we mention that we have verified up to $\mathcal{O}\left(t^{22}\right)$ (using computer algebra and higher order terms in (62) and (63), see appendix D) that the corrections to $\left\langle\delta g(t)^{2}\right\rangle$ in (67) are even in $t$ with positive coefficients. This shows explicitly that the non-analytic corrections indicated in (63) are vital for the onset of localization.

Now we have to generalize our treatment to the case $\Delta t>0$. We define a new variable $x$ by $\Delta t=t x$ and will only keep the lowest relevant order in $t$ but all orders in $x$. The expansions of $S_{1}(k, \Delta t)$ and $S_{2}(m, \Delta t)$ in powers of $\Delta t$ read (see appendix E)

$$
\begin{aligned}
S_{1}(k, \Delta t) & =\sum_{n=0}^{\infty} \frac{(-\Delta t)^{n}}{n !}(-1)^{n} r_{n+1}\left(-k^{2}\right) \\
S_{2}(m, \Delta t) & =\sum_{n=0}^{\infty} \frac{(-\Delta t)^{n}}{n !} r_{n+1}\left(\varepsilon_{m}\right)
\end{aligned}
$$

where $r_{n+1}(z)$ is a polynomial of degree $n+1$ in the variable $z$. In appendix E, we calculate the first three coefficients $a_{n}, b_{n}, c_{n}$ in

$$
r_{n+1}(z)=a_{n} z^{n+1}+b_{n} z^{n}+c_{n} z^{n-1}+\cdots
$$

with the result

$$
\begin{aligned}
& a_{n}=-\frac{1}{4(n+1)^{2}} \quad(n \geq 0), \\
& b_{n}= \begin{cases}\frac{(2 n+1)^{2}}{12 n(n+1)} & (n \geq 1) \\
\frac{1}{4} & (n=0)\end{cases} \\
& c_{n}= \begin{cases}-\frac{(2 n+1)(2 n-1)\left(12 n^{2}-5\right)}{180(n+1)(n-1)} & (n \geq 2) \\
-\frac{5}{16} & (n=1) \\
0 & (n=0)\end{cases}
\end{aligned}
$$


From (68)-(70) it is clear that we will need the following type of moments

$$
\begin{gathered}
{\left[\left(k^{2}\right)^{n}\right]_{t}^{(k)}=\left(-\partial_{t}\right)^{n}[1]_{t}^{(k)}=\frac{n !}{2 t^{n+1}}-\frac{1}{6} \delta_{n, 0}+\frac{7}{60}\left(\delta_{n, 0} t-\delta_{n, 1}\right)+\mathcal{O}\left(t^{2-n}\right),} \\
{\left[\varepsilon_{m}^{n}\right]_{t}^{(m)}=\left(-\partial_{t}\right)^{n}[1]_{t}^{(k)}=\frac{n !}{2 t^{n+1}}+\frac{1}{6} \delta_{n, 0}+\frac{7}{60}\left(\delta_{n, 0} t-\delta_{n, 1}\right)+\mathcal{O}\left(t^{2-n}\right),}
\end{gathered}
$$

which have been calculated using (62) and (63). Combining (68) and (69) with (72) and (73) we obtain

$$
\begin{gathered}
{\left[S_{1}(k, \Delta t)\right]_{t}^{(k)}=-\frac{1}{t^{2}} \hat{a}\left(\frac{\Delta t}{t}\right)+\frac{1}{t} \hat{b}\left(\frac{\Delta t}{t}\right)-\hat{c}\left(\frac{\Delta t}{t}\right)+\mathcal{O}(t)} \\
{\left[S_{2}(m, \Delta t)\right]_{t}^{(m)}=+\frac{1}{t^{2}} \hat{a}\left(\frac{\Delta t}{t}\right)+\frac{1}{t} \hat{b}\left(\frac{\Delta t}{t}\right)+\hat{c}\left(\frac{\Delta t}{t}\right)+\mathcal{O}(t)}
\end{gathered}
$$

with

$$
\begin{aligned}
\hat{a}(x) & =\frac{1}{2} \sum_{n=0}^{\infty}(n+1) a_{n}(-x)^{n}=-\frac{1}{8 x} \ln (1+x), \\
\hat{b}(x) & =\frac{1}{2} \sum_{n=0}^{\infty} b_{n}(-x)^{n}=\frac{1}{6(1+x)}-\frac{1}{24}\left(1+\frac{1}{x}\right) \ln (1+x), \\
\hat{c}(x) & =\frac{1}{6} b_{0}-\frac{7}{60} a_{0}+\frac{1}{2} \sum_{n=1}^{\infty} \frac{1}{n} c_{n}(-x)^{n} \\
& =\frac{1}{10}+\frac{7 x}{45}-\frac{4 x^{2}}{15(1+x)}+\frac{2 x^{3}}{15(1+x)^{2}}-\left(\frac{1}{72}+\frac{7}{240 x}+\frac{7 x}{240}\right) \ln (1+x) .
\end{aligned}
$$

With the help of the decomposition rule (59) we have

$$
\begin{aligned}
\left(-\partial_{t}\right)\langle\delta g(t) \delta g(t+\Delta t)\rangle= & \left\{\left[S_{1}(k, \Delta t)\right]_{t}^{(k)}-\left[e^{-k^{2} \Delta t}\right]_{t}^{(k, m)}[1]_{t}^{(k)}\right\}\left[e^{-\varepsilon_{m} \Delta t}\right]_{t}^{(m)} \\
& -\left\{\left[S_{2}(m, \Delta t)\right]_{t}^{(m)}+\left[e^{-\varepsilon_{m} \Delta t}\right]_{t}^{(k, m)}[1]_{t}^{(m)}\right\}\left[e^{-k^{2} \Delta t}\right]_{t}^{(k)}
\end{aligned}
$$

To proceed we need the following relations

$$
\begin{aligned}
{\left[e^{-k^{2} \Delta t}\right]_{t}^{(k)} } & =[1]_{t+\Delta t}^{(k)}=\frac{1}{2(t+\Delta t)}-\frac{1}{6}+\frac{7}{60}(t+\Delta t)+\cdots \\
{\left[e^{-\varepsilon_{m} \Delta t}\right]_{t}^{(m)} } & =[1]_{t+\Delta t}^{(m)}=\frac{1}{2(t+\Delta t)}+\frac{1}{6}+\frac{7}{60}(t+\Delta t)+\cdots \\
{\left[e^{-k^{2} \Delta t}\right]_{t}^{(k, m)} } & =-\int d t\left[e^{-k^{2} \Delta t}\right]_{t}^{(k)}[1]_{t}^{(m)} \\
& =\frac{1}{4} \ln \left(1+\frac{\Delta t}{t}\right)\left(\frac{1}{\Delta t}-\frac{1}{3}+\frac{7}{30} \Delta t\right)-\frac{4}{45}\left(t+\alpha_{1} \Delta t\right)+\ldots \\
{\left[e^{-\varepsilon_{m} \Delta t}\right]_{t}^{(k, m)} } & =\frac{1}{4} \ln \left(1+\frac{\Delta t}{t}\right)\left(\frac{1}{\Delta t}+\frac{1}{3}+\frac{7}{30} \Delta t\right)-\frac{4}{45}\left(t+\alpha_{2} \Delta t\right)+\ldots
\end{aligned}
$$

Here, $\alpha_{1}$ and $\alpha_{2}$ are two undetermined constants. However, adding (82) and (83) and expanding both sides for small $\Delta t$ we find the constraint $\alpha_{1}+\alpha_{2}=1$. It turns out that our 
final result does not depend on the single remaining unknown constant and we obtain from $(74)-(83)$

$$
\left(-\partial_{t}\right)\langle\delta g(t) \delta g(t+\Delta t)\rangle \simeq-\frac{2}{15} \frac{\Delta t}{t^{2}} \frac{1}{\left(1+\frac{\Delta t}{t}\right)^{3}}=-\frac{1}{15} \partial_{t}\left(\frac{1}{\left(1+\frac{\Delta t}{t}\right)^{2}}\right) .
$$

The result (58) follows immediately upon integrating this last relation. In principle we still have to worry about an integration constant that might depend on $\Delta t$. However, this constant must vanish for $\Delta t=0$ due to (67) and possible higher order terms are at least $\mathcal{O}(\Delta t)=\mathcal{O}(t)$

\section{LOCALIZED REGIME}

In this section we derive the fact that for $t=L / 2 \xi \gg 1$ (localized regime) but arbitrary channel number $N$ the conductance length correlation function depends exponentially on both $t$ and $\Delta t$.

In the limit $t \gg 1$ only the term with $m=0$ and the region of small $k$ contribute significantly to the expression (52). This observation simplifies (53) enormously. Since $[\ldots]_{t}^{(k, m)} \sim e^{-t}$ we can neglect higher powers of this symbol and with $S_{2}(0, \Delta t)=0$ we get

$$
\langle\delta g(t) \delta g(t+\Delta t)\rangle \simeq\left[S_{1}(k, \Delta t) e^{-\varepsilon_{m} \Delta t}\right]_{t}^{(k, m)} \simeq\left\langle\delta g^{2}(t)\right\rangle 4 S_{1}(0, \Delta t) e^{-\Delta t}
$$

Here, we have used the fact that $\left\langle\delta g(t)^{2}\right\rangle \approx \frac{1}{4}\left[k^{2}+\varepsilon_{m}\right]_{t}^{(k, m)} \approx \frac{1}{4}[1]_{t}^{(k, m)}$ (see (64)) in the localized limit. The average $\left\langle\delta g(t)^{2}\right\rangle$ can be calculated by means of a saddle-point approximation to give

$$
\left\langle\delta g^{2}(t)\right\rangle \simeq a(N, 0,0) \frac{\pi^{3 / 2}}{16} t^{-3 / 2} e^{-t}
$$

For $a(N, 0,0)$ we have from $(50)$

$$
a(N, 0,0)=\frac{\Gamma\left(N+\frac{1}{2}\right)^{2}}{\Gamma(N) \Gamma(N+1)}=\frac{\pi}{2} \frac{(2 N) !(2 N-1) !}{4^{2 N-1}[(N-1) ! N !]^{2}} .
$$

The function $4 S_{1}(0, \Delta t)$ can in general (i.e. for arbitrary $\Delta t$ ) only be evaluated numerically. In the limiting cases $\Delta t \gg 1$ and $\Delta t \ll 1$ the following approximations can be derived

$$
4 S_{1}(0, \Delta t) \simeq \begin{cases}1-\frac{5}{4} \Delta t+\frac{7}{4}(\Delta t)^{2}+\cdots & (\Delta t \ll 1) \\ I(0,0) \frac{\pi^{3 / 2}}{4}(\Delta t)^{-3 / 2} & (\Delta t \gg 1)\end{cases}
$$

where $I(0,0)=14 \zeta(3) / \pi^{2} \simeq 1.705$ is given by the integral (56) at $k=\tilde{k}=0$. A particular consequence of our results is the very symmetric expression

$$
\langle\delta g(t) \delta g(t+\Delta t)\rangle=\text { const. }(\Delta t)^{-3 / 2} t^{-3 / 2} e^{-\Delta t} e^{-t}
$$

for $t, \Delta t \gg 1$. 


\section{CROSSOVER REGIME}

To describe the crossover behavior of the conductance length correlation function between the metallic and the localized regime (in both $t$ and $\Delta t$ ) we have to rely on a numerical evaluation of (53). We have already mentioned earlier that the key quantity $I(k, \tilde{k})(56)$ is difficult to calculate as it stands and that in appendix $\mathrm{C}$ a more suitable expression (as far as a numerical treatment is concerned) is derived.

In all the computations reported here we have set the channel number $N$ to infinity. Let us define the quantity

$$
K(t, \Delta t)=\frac{\langle\delta g(t) \delta g(t+\Delta t)\rangle}{\langle\delta g(t) \delta g(t)\rangle}\left(1+\frac{\Delta t}{t}\right)^{2} .
$$

Obviously, this is the conductance length correlation function normalized to its value at $\Delta t=0$ and multiplied by the inverse of the squared Lorentzian (58) to compensate for the "trivial" metallic behavior. By definition, $K(t, \Delta t)$ should be a constant equal to unity for $t \ll 1$ and not too large $\Delta t$. As $\Delta t$ increases localization effects must show up and for large $\Delta t \gg 1$ we expect an exponential decay of $K(t=$ const. $\ll 1, \Delta t)$. For increasing $t$, on the other hand, we expect the compensation factor $(1+\Delta t / t)^{2}$ to become ineffective even for small $\Delta t$ since $K(t=$ const, $\Delta t)$ should decay exponentially rather than algebraically for $t \gg 1$. In Fig. 1 we have plotted $K(t, \Delta t)$ as a function of $\Delta t$ for $t=0.1,0.2,0.5,1.0$, and 5.0. Clearly, our expectations are borne out. However, there is one intriguing and unforeseen feature: The curve $K(t=0.1, \Delta t)$ bears as function of $\Delta t$ striking similarities to the behavior of the variance $\langle\delta g(t) \delta g(t)\rangle$ of the conductance in the unitary case. This observation suggests that (58) remains true if we replace the numerical prefactor $1 / 15$ by the full function $\langle\delta g(\Delta t) \delta g(\Delta t)\rangle$, i.e.

$$
\langle\delta g(t) \delta g(t+\Delta t)\rangle=\frac{\langle\delta g(\Delta t) \delta g(\Delta t)\rangle}{\left(1+\frac{\Delta t}{t}\right)^{2}}+\mathcal{O}(t)
$$

We have not tried to prove this relation analytically and a numerical verification turns out to be difficult since we cannot go easily to $t$ values smaller than 0.1. Therefore (91) remains an interesting speculation for the time being.

Next, to characterize the distance over which two conductances are correlated, we have determined the point $(\Delta t)_{1 / e}$ such that $\left\langle\delta g(t) \delta g\left(t+(\Delta t)_{1 / e}\right)\right\rangle=\langle\delta g(t) \delta g(t)\rangle / e$. In Fig. 2 we have plotted $(\Delta t)_{1 / e}$ as a function of $t$. We see that the curve starts with a linear behavior $(\Delta t)_{1 / e} \sim t$ for $t<1$ and then saturates at $(\Delta t)_{1 / e} \approx 0.5$ for $t \gg 1$. This is exactly what we expect from the limiting cases worked out in sections V and VI. In the metallic regime the decay scale is set by the system length itself, see (58). This is also dictated by the universality of the conductance fluctuations: There can be no intrinsic length scale other than the system length. In the localized regime, however, we have the asymptotic expression (89) and the situation changes. The exponential function, of course, does decay on a typical

intrinsic scale. Since $\Delta t=\Delta L / 2 \xi$ and the curve in Fig. 2 saturates at $(\Delta t)_{1 / e} \approx 0.5$ we see explicitly that the wire is divided into independent segments of size $\xi$. 


\section{SUMMARY AND CONCLUSIONS}

Employing techniques that have recently been developed to calculate the transport properties of a quasi 1d wire in the Fokker-Planck approach we have derived the expression (53) for the conductance length correlation function $\langle\delta g(t) \delta g(t+\Delta t)\rangle$. In the metallic regime $(t \ll 1, \Delta t<1, N \rightarrow \infty)$ we found a squared Lorentzian

$$
\langle\delta g(t) \delta g(t+\Delta t)\rangle=\frac{1}{15} \frac{1}{\left(1+\frac{\Delta t}{t}\right)^{2}}+\mathcal{O}(t)
$$

while in the localized regime $(t, \Delta t \gg 1)$ the correlation function is dominated by exponential tails,

$$
\langle\delta g(t) \delta g(t+\Delta t)\rangle=\text { const. }(\Delta t)^{-3 / 2} t^{-3 / 2} e^{-\Delta t} e^{-t} .
$$

For intermediate values of $t$ and $\Delta t$ we had to restrict ourselves to a numerical evaluation of the various sums and integrals in (53). Figs. 1 and 2 show the crossover from the metallic to the localized regime both in the typical dependence on $\Delta t$ and in the dependence of the correlation width $(\Delta t)_{1 / e}$ on $t$. The latter quantity is proportional to $t$ in the metallic regime and saturates at $(\Delta t)_{1 / e} \approx 0.5$ (i.e. $\left.\Delta L \approx \xi\right)$ in the localized regime.

Eq.(58) generalizes the celebrated universality of the conductance fluctuations to the case of the length correlation function. As long as the ratio $\Delta t / t$ is constant the absolute length of the system does not matter. The result also shows that any rearrangement in the wire (of the disorder potential, say) affects the full system since the correlation width is given by the system length. Fig. 2 proves that this changes drastically in the localized case. As the system size exceeds the localization length $\xi$, the correlation length no longer grows with $t$ but saturates at $\Delta L \approx \xi$. This demonstrates explicitly that the wire is decomposed into statistically independent segments of size $\xi$. As a consequence, moving one impurity no longer changes the statistical properties of the whole wire but affects only the relevant segment. From this result one can deduce [8] that the conductance fluctuations $\left\langle\left[\ln g / g^{\prime}\right]^{2}\right\rangle$ in a quasi 1d wire in the localized regime are universal and of order unity.

Acknowledgements. We thank Jean-Louis Pichard for several helpful remarks. K.F. acknowledges C.W.J. Beenakker for fruitful discussions. This work was supported by the DFG and the European HCM program (K.F.), and a NATO fellowship through the DAAD (A.M.G.). 


\section{FIGURES}

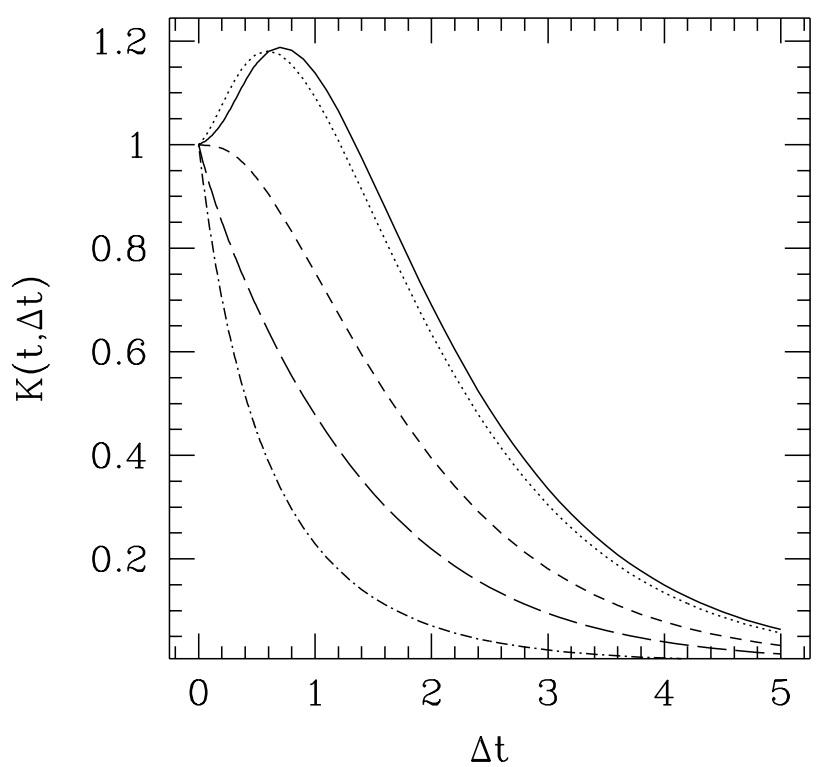

FIG. 1. The function $K(t, \Delta t)$ as defined in the text versus $\Delta t$. The curves correspond from top to bottom to $t=0.1$ (solid), $t=0.2$ (dotted), $t=0.5$ (dashed), $t=1.0$ (long dashes), and $t=5.0$ (dash-dotted).

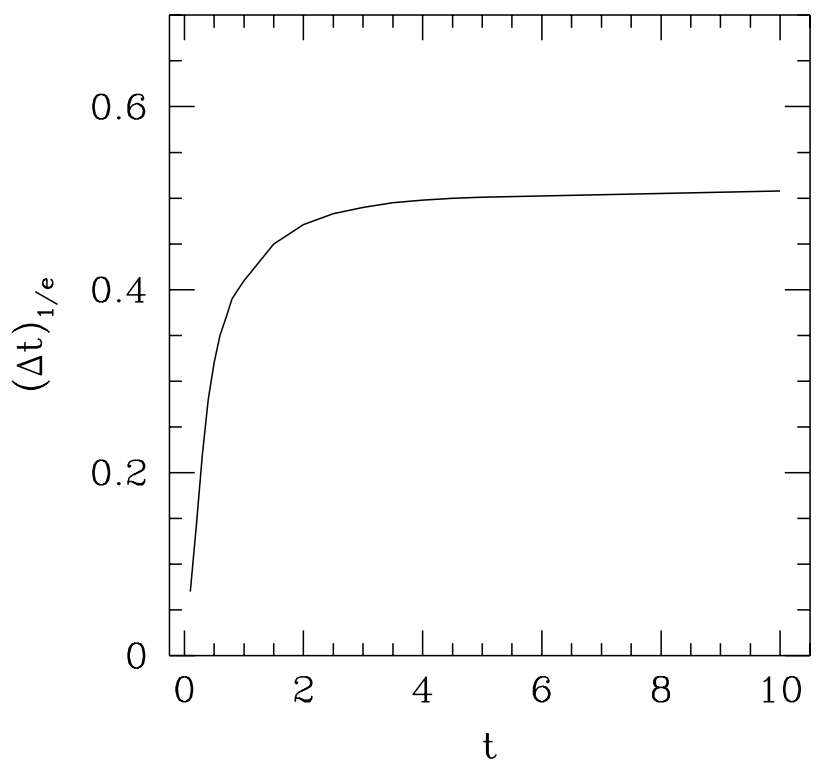

FIG. 2. The decay width $(\Delta t)_{1 / e}$ of the length correlation function versus the system length $t$. 


\section{APPENDIX A: PROOF OF (40)}

From the definition (13) it is clear that we have to demonstrate that

$$
\frac{1}{1+\lambda} P_{m}(1+2 \lambda)=(-1)^{m}\left(\frac{1}{1+\lambda}+\sum_{l=0}^{m-1} a_{m l} P_{l}(1+2 \lambda)\right)
$$

in order to prove (40). Since $P_{m}(1+2 \lambda)$ is a polynomial of degree $m$ in the variable $1+\lambda$ an expansion of the form (A1) must exist. The coefficients $a_{m l}$ can be calculated as follows. With the help of the orthogonality relation for the Legendre polynomials, $\int_{-1}^{1} d x P_{n}(x) P_{m}(x)=\delta_{n m} 2 /(1+2 n)$, we get for the $a_{m l}(x$ corresponds to $1-2(1+\lambda))$

$$
a_{m l}=(-1)^{l}(1+2 l) \int_{-1}^{1} d x \frac{1}{1-x}\left(P_{m}(x)-1\right) P_{l}(x)
$$

This integral vanishes for $m \leq l$. For $m>l$ and using $\left(P_{m}(x)-1\right) P_{l}(x)=\left(P_{m}(x)-1\right)-$ $\left(P_{l}(x)-1\right)+P_{m}(x)\left(P_{l}(x)-1\right)$ we find

$$
a_{m}=(-1)^{l}(1+2 l)\left(b_{m}-b_{l}\right)
$$

with

$$
b_{m}=\int_{-1}^{1} d x \frac{P_{m}(x)-1}{1-x}
$$

Applying the recurrence relation for the Legendre polynomials, $(2 m+1) x P_{m}(x)=$ $(m+1) P_{m+1}(x)+m P_{m-1}(x)$, we obtain the equation

$$
b_{m+1}-b_{m}=-2 \delta_{m, 0}+\frac{m}{m+1}\left(b_{m}-b_{m-1}\right)
$$

which has the solution

$$
b_{m}=-2 \sum_{\nu=1}^{m} \frac{1}{\nu}
$$

Together with (A2) this gives precisely the value for $a_{m l}$ claimed in (40).

\section{APPENDIX B: THE INTEGRAL (49)}

In this appendix, we calculate the integral (49) for arbitrary complex values of $\alpha$ with $\operatorname{Re}(\alpha)>\frac{1}{2}$. Using (10) and formula 15.3.4 of Ref. [13] we get

$$
\psi_{k}(\lambda)=F\left(\frac{1}{2}-i \frac{k}{2}, \frac{1}{2}+i \frac{k}{2} ; 1 ;-\lambda\right)=(1+\lambda)^{(-1+i k) / 2} F\left(\frac{1}{2}-i \frac{k}{2}, \frac{1}{2}-i \frac{k}{2} ; 1 ; \frac{\lambda}{1+\lambda}\right) \quad .
$$

Substituting $s=(1+\lambda)^{-1}$ and expanding the hypergeometric series the l.h.s. of (49) becomes

$$
\sum_{n=0}^{\infty} \frac{\left(\frac{1}{2}-i \frac{k}{2}\right)_{n}^{2}}{n !^{2}} \int_{0}^{1} d s s^{-3 / 2-i k / 2+\alpha}(1-s)^{n}=\sum_{n=0}^{\infty} \frac{\left(\frac{1}{2}-i \frac{k}{2}\right)_{n}^{2}}{n !^{2}} \frac{\Gamma\left(-\frac{1}{2}-i \frac{k}{2}+\alpha\right) \Gamma(n+1)}{\Gamma\left(\frac{1}{2}-i \frac{k}{2}+\alpha+n\right)}
$$


Here, $(a)_{n}=a(a+1) \cdots(a+n-1)=\Gamma(a+n) / \Gamma(a)$ is the Pochhammer symbol [13]. The evaluation of the integral involves the $\beta$-function [13]. The sum in (B2) can be expressed as a certain hypergeometric function at the special value $z=1$ and we can write for the r.h.s. of (B2):

$$
\frac{\Gamma\left(-\frac{1}{2}-i \frac{k}{2}+\alpha\right)}{\Gamma\left(\frac{1}{2}-i \frac{k}{2}+\alpha\right)} \sum_{n=0}^{\infty} \frac{\left(\frac{1}{2}-i \frac{k}{2}\right)_{n}^{2}}{n !\left(\frac{1}{2}-i \frac{k}{2}+\alpha\right)_{n}}=\frac{\Gamma\left(-\frac{1}{2}-i \frac{k}{2}+\alpha\right)}{\Gamma\left(\frac{1}{2}-i \frac{k}{2}+\alpha\right)} F\left(\frac{1}{2}-i \frac{k}{2}, \frac{1}{2}-i \frac{k}{2} ; \frac{1}{2}-i \frac{k}{2}+\alpha ; 1\right) .
$$

Using formula 15.1 .20 of Ref. [13] we finally obtain the r.h.s. of Eq. (49).

\section{APPENDIX C: THE INTEGRAL (56)}

We derive an expression for $I(k, \tilde{k})$ defined in (56) that is suitable for a numerical evaluation. Applying the transformation formula 15.3.8 of Ref. [13] we have

$$
\psi_{k}(\mu)=\frac{\Gamma(i k)}{\Gamma\left(\frac{1}{2}+i \frac{k}{2}\right)^{2}}(1+\mu)^{(-1+i k) / 2} F\left(\frac{1}{2}-i \frac{k}{2}, \frac{1}{2}-i \frac{k}{2} ; 1-i k ; \frac{1}{1+\mu}\right)+(k \leftrightarrow-k) .
$$

We expand the hypergeometric function in (C1) and replace the first $\psi_{k}(\mu)$ in (56) by the resulting sum. For each term in the sum the $\mu$-integration can be done by using the integral (49) derived in the previous appendix. After some simplification the result for $I(k, \tilde{k})$ reads:

$$
\begin{aligned}
I(k, \tilde{k})= & \frac{\Gamma(i k) \Gamma\left(1-\frac{i}{2}(k+\tilde{k})\right) \Gamma\left(1-\frac{i}{2}(k-\tilde{k})\right)}{\Gamma\left(\frac{1}{2}+i \frac{k}{2}\right)^{2} \Gamma\left(\frac{1}{2}-i \frac{k}{2}\right)^{2}} \\
& \times \sum_{n=0}^{\infty} \frac{\left(1-\frac{i}{2}(k+\tilde{k})\right)_{n}\left(1-\frac{i}{2}(k-\tilde{k})\right)_{n}}{n !(1-i k)_{n}} \frac{1}{\left(\frac{1}{2}-i \frac{k}{2}+n\right)^{2}}+(k \leftrightarrow-k) .
\end{aligned}
$$

We cannot proceed along the lines of appendix B to evaluate the sum in (C2) due to the presence of the extra factor $(z+n)^{-2}$ (with $z=\frac{1}{2}-i \frac{k}{2}$ ). A direct numerical summation, on the other hand, is inefficient since the sum converges only like $n^{-2}$. However, the convergence can be substantially improved by the expansion

$$
\frac{1}{(z+n)^{2}}=\sum_{\nu=2}^{m} \frac{C_{\nu}(z)}{(2 z+n)_{\nu}}+\frac{A_{m}(z)}{(z+n)^{2}(2 z+n)_{m-1}}+\frac{B_{m}(z)}{(z+n)^{2}(2 z+n)_{m}} .
$$

The coefficients $C_{\nu}(z), A_{m}(z), B_{m}(z)$ depend on $z$ but not on $n$ and can be determined by a suitable recurrence relation. The contributions involving $C_{\nu}(z)$ can be summed analytically by identifying (as in appendix B) a hypergeometric function $F(., . ; . ; 1)$. The remaining terms with $A_{m}(z)\left(B_{m}(z)\right)$ behave as $\sim n^{-(1+m)}\left(\sim n^{-(2+m)}\right)$. Choosing $m=10-20$ the corresponding sums converge rapidly. 


\section{APPENDIX D: EXPANSION OF (62) AND 63)}

This appendix deals with the expansion (in powers of $t$ ) of

$$
\begin{aligned}
& {[1]_{t}^{(k)}=\int_{0}^{\infty} d k k \tanh \left(\frac{\pi k}{2}\right) e^{-k^{2} t}} \\
& {[1]_{t}^{(m)}=\sum_{0}^{\infty} 2(1+2 m) e^{-(1+2 m)^{2} t}}
\end{aligned}
$$

in the limit $t \ll 1$. Expanding $\tanh \left(\frac{\pi k}{2}\right)$ in terms of exponential functions we obtain:

$$
\begin{aligned}
{[1]_{t}^{(k)} } & =\int_{0}^{\infty} d k k\left(1-2 \sum_{n=0}^{\infty}(-1)^{n} e^{-(1+n) \pi k}\right) e^{-k^{2} t} \\
& =\frac{1}{2 t}-2 \sum_{n=0}^{\infty}(-1)^{n} \int_{0}^{\infty} d k k e^{-(1+n) \pi k} \sum_{m=0}^{\infty} \frac{(-1)^{m} t^{m}}{m !} k^{2 m} \\
& =\frac{1}{2 t}-2 \sum_{m=0}^{\infty} \frac{(-1)^{m} t^{m}}{m !} \frac{(1+2 m) !}{\pi^{2(m+1)}} \sum_{n=0}^{\infty}(-1)^{n} \frac{1}{(n+1)^{2(m+1)}} \\
& =\frac{1}{2 t}-2 \sum_{m=0}^{\infty} \frac{(-1)^{m} t^{m}}{m !} \frac{(1+2 m) !}{\pi^{2(m+1)}}\left(1-\frac{1}{\left.2^{2 m+1}\right) \zeta(2 m+2)}\right. \\
& =\frac{1}{2 t}\left(1+\sum_{m=1}^{\infty}\left[\frac{(-1)^{m} 2\left(2^{2 m-1}-1\right)}{m !}\left|B_{2 m}\right|\right] t^{m}\right) .
\end{aligned}
$$

In the last step we have used that $\zeta(2 m)=(2 \pi)^{2 m}\left|B_{2 m}\right| /[2(2 m) !]$, where the $B_{2 m}$ denote the Bernoulli numbers [13. With $B_{2}=\frac{1}{6}$ and $B_{4}=-\frac{1}{30}$ we find the first three terms in (62).

In order to treat the discrete sum (D2) we decouple the quadratic term in the exponent by a Gaussian integral:

$$
\begin{aligned}
{[1]_{t}^{(m)} } & =\lim _{\eta \rightarrow 0+} \frac{1}{\sqrt{\pi t}} \int_{-\infty}^{\infty} d s e^{-s^{2} / t} \sum_{m=0}^{\infty} 2(1+2 m) e^{(1+2 m)(2 i s-\eta)} \\
& =\lim _{\eta \rightarrow 0+} \frac{1}{\sqrt{\pi t}} \int_{-\infty}^{\infty} d s e^{-s^{2} / t} \frac{1}{2} \partial_{s}\left(\frac{1}{\sin (2 s+i \eta)}\right) \\
& =\lim _{\eta \rightarrow 0+} \frac{1}{2 t \sqrt{\pi t}} \int_{-\infty}^{\infty} d s \frac{2 s}{\sin (2 s+i \eta)} e^{-s^{2} / t} .
\end{aligned}
$$

In the limit $\eta \rightarrow 0+$, the integrand has $\delta$-function contributions at $s=\frac{\pi}{2} n$, where $n \neq 0$ is an integer. These lead to the non-analytic corrections $\sim e^{-\pi^{2} n^{2} /(4 t)}$, which we will neglect in the following. The analytic corrections arise from small $s$-contributions in the integral. Expanding $(2 s) / \sin (2 s)$ in a power series (formula 4.3 .68 of Ref. [13]) and evaluating the Gaussian integrals we arrive at:

$$
[1]_{m}^{(t)}=\frac{1}{2 t} \sum_{m=0}^{\infty}\left[\frac{(-1)^{m-1} 2\left(2^{2 m-1}-1\right)}{m !} B_{2 m}\right] t^{m}+\mathcal{O}\left(e^{-\pi^{2} /(4 t)}\right)
$$

Remarkably, the coefficients differ only by the sign $(-1)^{m}$ from those in (D3). Again, the first three terms yield (63). 


\section{APPENDIX E: CALCUATION OF THE COEFFICIENTS (71)}

In order to expand $S_{1}(k, \Delta t)$ we exploit the fact that the $\lambda$-integration in (54) can be viewed as the formal application of the operator $\exp [-D(\mu) \Delta t]$ on $(1+\mu)^{-1}$ (with $D(\mu)$ as in $(8))$ :

$$
\begin{aligned}
\int_{0}^{\infty} d \lambda g(\mu, \lambda ; \Delta t) \frac{1}{1+\lambda} & =e^{-D(\mu) \Delta t}\left(\frac{1}{1+\mu}\right)=\sum_{n=0}^{\infty} \frac{(\Delta t)^{n}}{n !}[-D(\mu)]^{n}\left(\frac{1}{1+\mu}\right) \\
& =\sum_{n=0}^{\infty} \frac{(\Delta t)^{n}}{n !} R_{n}(\mu)
\end{aligned}
$$

Here, $R_{n}(\mu)=[-D(\mu)]^{n}(1+\mu)^{-1}$ is a polynomial of degree $n+1$ in $(1+\mu)^{-1}$, i.e. we may write

$$
R_{n}(\mu)=\sum_{l=0}^{n} r_{n, l}(1+\mu)^{-(l+1)},
$$

where the coefficient $r_{n, l}$ obeys the recurrence relation

$$
r_{n+1, l}=-4 l^{2} r_{n, l-1}+(2 l+1)^{2} r_{n, l}
$$

with $r_{0, k}=\delta_{0, k}$. To calculate the coefficients $a_{n}, b_{n}, c_{n}$ in (70), we need the values of $r_{n, n}$, $r_{n, n-1}$ and $r_{n, n-2}$. From (E3) we find

$$
\begin{aligned}
r_{n, n} & =(-4)^{n} n !^{2} \\
r_{n, n-1} & =(-4)^{n-1}(n-1) !^{2} \sum_{\nu=0}^{n-1}(1+2 \nu)^{2}, \\
r_{n, n-2} & =(-4)^{n-2}(n-2) !^{2} \sum_{\nu=0}^{n-2}(1+2 \nu)^{2} \sum_{\tilde{\nu}=0}^{\nu}(1+2 \tilde{\nu})^{2}
\end{aligned}
$$

On the other hand, we may insert (E1) and (E2) in (54) and perform the $\mu$-integration using (49), giving

$$
S_{1}(k, \Delta t)=\sum_{n=0}^{\infty} \frac{(\Delta t)^{n}}{n !} \sum_{l=0}^{n} r_{n, l} \frac{1}{(l+1) !^{2}} \prod_{\nu=0}^{l}\left(\left(\frac{1}{2}+\nu-i \frac{k}{2}\right)\left(\frac{1}{2}+\nu+i \frac{k}{2}\right)\right)
$$

The product is a polynomial of degree $(l+1)$ in $k^{2}$, of which we need the first three terms:

$$
\begin{aligned}
\prod_{\nu=0}^{l}\left(\left(\frac{1}{2}+\nu-i \frac{k}{2}\right)\left(\frac{1}{2}+\nu+i \frac{k}{2}\right)\right) & =\frac{1}{4^{l+1}} \prod_{\nu=0}^{l}\left(k^{2}+(1+2 \nu)^{2}\right) \\
& =\frac{1}{4^{l+1}}\left(\left(k^{2}\right)^{l+1}+A_{l}\left(k^{2}\right)^{l}+B_{l}\left(k^{2}\right)^{l-1}+\cdots\right)
\end{aligned}
$$

with 


$$
\left.A_{l}=\sum_{\nu=0}^{l}(1+2 \nu)^{2} \quad, \quad B_{l}=\sum_{0 \leq \nu<\tilde{\nu} \leq l}(1+2 \nu)^{2} 1+2 \tilde{\nu}\right)^{2} .
$$

Together, (E6) and (E7) imply

$$
\begin{aligned}
& a_{n}=\frac{(-1)^{n+1}}{4^{n+1}(n+1) !^{2}} r_{n, n}, \\
& b_{n}=\frac{(-1)^{n+1}}{4^{n+1}(n+1) !^{2}} r_{n, n} A_{n}+\frac{(-1)^{n}}{4^{n} n !^{2}} r_{n, n-1}, \\
& c_{n}=\frac{(-1)^{n+1}}{4^{n+1}(n+1) !^{2}} r_{n, n} B_{n}+\frac{(-1)^{n}}{4^{n} n !^{2}} r_{n, n-1} A_{n}+\frac{(-1)^{n-1}}{4^{n-1}(n-1) !^{2}} r_{n, n-2} .
\end{aligned}
$$

for the desired coefficients. Combining (E4), (E8) and (E9), one indeed obtains after some (computer-)algebra the result (71). It remains to show that $S_{2}(m, \Delta t)$ is given by (69) with the same coefficients (71). Expanding (55) we find:

$$
S_{2}(m, \Delta t)=\sum_{n=0}^{\infty} \frac{(-\Delta t)^{n}}{n !}(-2) C_{n}(m)
$$

with

$$
C_{n}(m)=\sum_{\nu=1}^{m} \frac{1}{\nu} \sum_{l=0}^{\nu-1}(1+2 l)^{1+2 n} .
$$

For small $n$ the $C_{n}(m)$ can be calculated with the help of standard mathematical formulas. For our purposes, however, we need the large $-m$ behavior for all $n$. Therefore we consider the generating function

$$
g(m, x)=\sum_{\nu=1}^{m} \frac{1}{\nu} \sum_{l=0}^{\nu-1} \sinh [(1+2 l) x]=\sum_{n=0}^{\infty} \frac{x^{1+2 n}}{(1+2 n) !} C_{n}(m)
$$

Using (twice) the finite geometric series we can derive the inhomogeneous differential equation

$$
\sinh (2 x) g(m, x)+(\cosh (2 x)-1) \partial_{x} g(m, x)=\cosh [(1+2 m) x]-\cosh x,
$$

from which we get the recurrence relation

$$
C_{n}(m)=\frac{1}{b_{n n}}\left(\varepsilon_{m}^{n+1}-1-\sum_{k=0}^{n-1} b_{n k} C_{k}(m)\right)
$$

with

$$
b_{n k}=\frac{n+k+2}{2 n+3}\left(\begin{array}{c}
2 n+3 \\
2 k+1
\end{array}\right) 2^{2(n-k+1)} .
$$

Eq. (E14) indeed gives after some algebra 


$$
(-2) C_{n}(m)=a_{n} \varepsilon_{m}^{n+1}+b_{n} \varepsilon_{m}^{n}+c_{n} \varepsilon_{m}^{n-1}+\cdots
$$

with $a_{n}, b_{n}, c_{n}$ as in (71).

We conclude this appendix with a short remark: Equations (68) and (69) suggest that the functions $S_{1}(k, \Delta t)$ and $S_{2}(m, \Delta t)$ are connected by the analytic replacement $-k^{2} \rightarrow$ $\varepsilon_{m}=(1+2 m)^{2}$ and $\Delta t \rightarrow-\Delta t$. We have verified this for the lowest $(n \leq 2)$ polynomials $r_{n+1}(z)$ and - as far as the highest coefficients $a_{n}, b_{n}, c_{n}$ appearing in (70) are concerned for all $n$. This is sufficient for the purpose of the present paper. A general proof, however, has yet to be given. 


\section{REFERENCES}

[1] K. B. Efetov and A. I. Larkin, Zh. Eksp. Theor. Fiz. 85, 764 (1983); Sov. Phys. JETP 58, 444 (1983).

[2] O. N. Dorokhov, Pis'ma Zh. Eksp. Teor. Fiz. 36, 259 (1982) [JETP Lett. 36, 318 (1982)].

[3] P. A. Mello, P. Pereyra, and. N. Kumar, Ann. Phys. (N.Y.) 181, 290 (1988).

[4] P. W. Brouwer and K. Frahm, Phys. Rev. B 53, 1490 (1996).

[5] M. R. Zirnbauer, Phys. Rev. Lett. 69, 1584 (1992); A. D. Mirlin, A. Müller-Groeling, and M.R. Zirnbauer, Ann. Phys. (N.Y.) 236, 325 (1994).

[6] C. W. J. Beenakker and B. Rejaei, Phys. Rev. Lett. 71, 3689 (1993); C. W. J. Beenakker and B. Rejaei, Phys. Rev. B 49, 7499 (1994); B. Rejaei, Thesis 1994, University of Leiden.

[7] K. Frahm, Phys. Rev. Lett. 74, 4706 (1995); K. Frahm and J.-L. Pichard, J. Phys. I France 5, 877 (1995).

[8] S. Feng, J.-L. Pichard, and F. Zeng, Phys. Rev. B48, 2529 (1993).

[9] P. A. Mello, Phys. Rev. Lett. 60, 1089 (1988).

[10] P. A. Mello and A. D. Stone, Phys. Rev. B 44, 3559 (1991).

[11] A. M. S. Macedo and J. T. Chalker, Phys. Rev. B 46, 14985 (1992).

[12] A. D. Stone, P. A. Mello, K. Muttalib, and J.-L. Pichard in Mesoscopic Phenomena in Solids, edited by B. L. Altschuler, P. A. Lee, and R. A. Webb (North Holland, Amsterdam, 1991).

[13] M. Abramowitz and I.A. Stegun, Handbook of Mathematical Functions, National Bureau of Standards, Washington 1964.

[14] M. L. Mehta, Random Matrices, 2nd ed. (Academic, New York, 1991). 